\title{
DYNAMICALLY GENERATED BARYON RESONANCES
}

\author{
M.F.M. LUTZ AND J. HOFMANN \\ Gesellschaft für Schwerionenforschung (GSI) \\ Planck Str. 1, 64291 Darmstadt, Germany
}

\begin{abstract}
Identifying a zero-range exchange of vector mesons as the driving force for the s-wave scattering of pseudo-scalar mesons off the baryon ground states, a rich spectrum of molecules is formed. We argue that chiral symmetry and large- $N_{c}$ considerations determine that part of the interaction which generates the spectrum. We suggest the existence of strongly bound crypto-exotic baryons, which contain a charm-anti-charm pair. Such states are narrow since they can decay only via OZI-violating processes. A narrow nucleon resonance is found at mass $3.52 \mathrm{GeV}$. It is a coupled-channel bound state of the $\left(\eta_{c} N\right),\left(\bar{D} \Sigma_{c}\right)$ system, which decays dominantly into the $\left(\eta^{\prime} N\right)$ channel. Furthermore two isospin singlet hyperon states at mass $3.23 \mathrm{GeV}$ and $3.58 \mathrm{GeV}$ are observed as a consequence of coupled-channel interactions of the $\left(\bar{D}_{s} \Lambda_{c}\right),\left(\bar{D} \Xi_{c}\right)$ and $\left(\eta_{c} \Lambda\right),\left(\bar{D} \Xi_{c}^{\prime}\right)$ states. Most striking is the small width of about $1 \mathrm{MeV}$ of the lower state. The upper state may be significantly broader due to a strong coupling to the $\left(\eta^{\prime} \Lambda\right)$ state. The spectrum of crypto-exotic charm-zero states is completed with an isospin triplet state at $3.93 \mathrm{GeV}$ and an isospin doublet state at $3.80 \mathrm{GeV}$. The dominant decay modes involve again the $\eta^{\prime}$ meson.
\end{abstract}

\section{Introduction}

The existence of strongly bound crypto-exotic baryon systems with hidden charm would be a striking feature of strong interactions ${ }^{1,2,3}$. Such states may be narrow since their strong decays are OZI-suppressed ${ }^{4}$. There are experimental hints that such states may indeed be part of nature. A high statistics bubble chamber experiment performed 30 years ago with a $K^{-}$ beam reported on a possible signal for a hyperon resonance of mass 3.17 $\mathrm{GeV}$ of width smaller than $20 \mathrm{MeV}^{5}$. About ten years later a further bubble chamber experiment using a high energy $\pi^{-}$beam suggested a nucleon resonance of mass $3.52 \mathrm{GeV}$ with a narrow width of $7_{-7}^{+20} \mathrm{MeV}$. In Fig. 1 we recall the measured five body $\left(p K^{+} K^{0} \pi^{-} \pi^{-}\right)$invariant mass distribution ${ }^{6}$ suggesting the existence of a crypto exotic nucleon resonance.

It is the purpose of the present talk to review a study addressing the possible existence of crypto-exotic baryon systems ${ }^{7}$. In view of the highly 


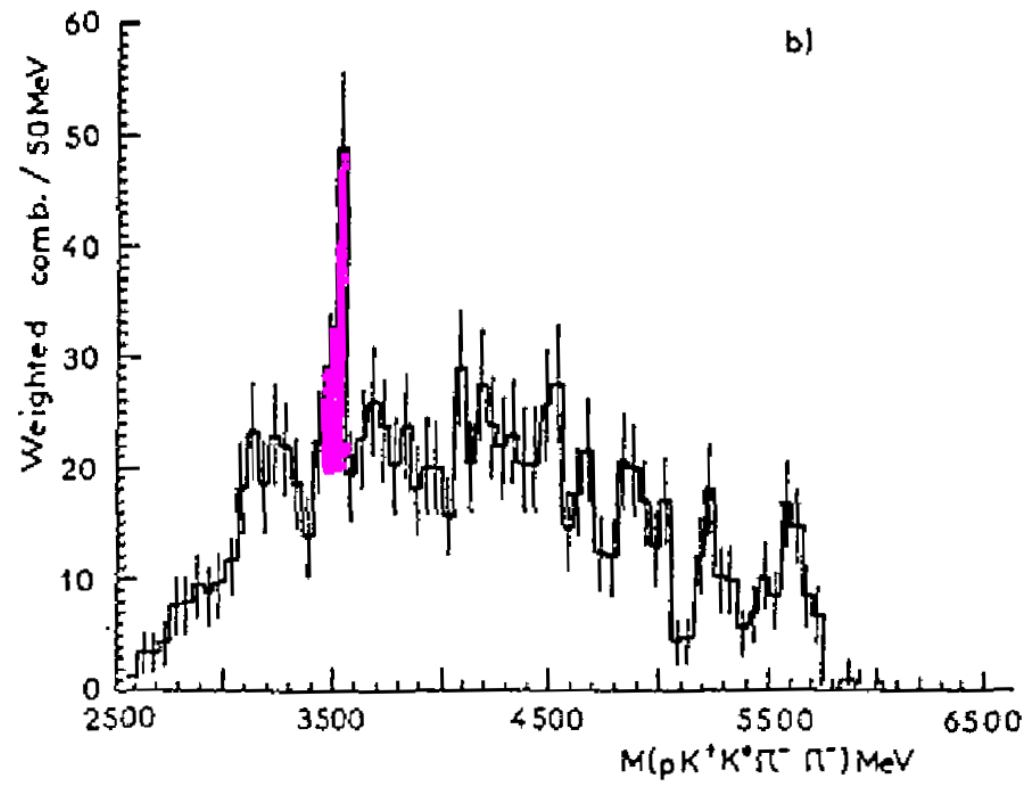

Figure 1. Measured five-body invariant mass distribution in a $19 \mathrm{GeV} \pi^{-}$beam experiment at CERN. The figure is taken from Ref. ${ }^{6}$

speculative nature of such states it is important to correlate the properties of such states to those firmly established, applying a unified and quantitative framework. We extended previous works ${ }^{8,9}$ that performed a coupledchannel study of the s-wave scattering processes where a Goldstone boson hits an open-charm baryon ground state. The spectrum of $J^{P}=\frac{1}{2}^{-}$and $J^{P}=\frac{3}{2}^{-}$molecules obtained in ${ }^{8,9}$ is quite compatible with the so far very few observed states. Analogous computations successfully describe the spectrum of open-charm mesons with $J^{P}=0^{+}$and $1^{+}$quantum numbers 10,11. These developments were driven by the hadrogenesis conjecture: meson and baryon resonances that do not belong to the large- $N_{c}$ ground state of QCD should be viewed as hadronic molecular states ${ }^{12,13,14,15,16}$. Generalizing those computations to include $\mathrm{D}$ - and $\eta_{c}$-mesons in the intermediate states offers the possibility to address the formation of crypto-exotic baryon states (see also ${ }^{17,18,19,20}$ ).

The results of ${ }^{8,9}$ were based on the leading order chiral Lagrangian, 
that predicts unambiguously the s-wave interaction strength of Goldstone bosons with open-charm baryon states in terms of the pion decay constant. Including the light vector mesons as explicit degrees of freedom in a chiral Lagrangian gives an interpretation of the leading order interaction in terms of the zero-range t-channel exchange of light vector mesons ${ }^{21,22,23,24,25}$. The latter couple universally to any matter field in this type of approach. Based on the assumption that the interaction strength of D- and $\eta_{c}$-mesons with the baryon ground states is also dominated by the t-channel exchange of the light vector mesons, we performed a coupled-channel study of cryptoexotic baryon resonances ${ }^{7}$.

\section{Coupled-channel interactions}

We consider the interaction of pseudoscalar mesons with the ground-state baryons composed out of u,d,s,c quarks. The pseudoscalar mesons that are considered in this work can be grouped into multiplet fields $\Phi_{[9]}, \Phi_{[\overline{3}]}$ and $\Phi_{[1]}$ corresponding to the Goldstone bosons, the $\eta^{\prime}$, the $D$-mesons and the $\eta_{c}$. The baryon states are collected into SU(3) multiplet fields $B_{[8]}, B_{[6]}$ and $B_{[\overline{3}]}$ with charm 0,1 and 1 . For an explicit representation of the various multiplet fields we refer to ${ }^{7}$.

In a first step we construct the interaction of the mesons and baryon fields with the nonet-field $V_{[9]}^{\mu}$ of light vector mesons. We write down a list of relevant SU(3) invariant 3-point vertices that involve the minimal number of derivatives. Consider first the terms involving pseudo-scalar fields:

$$
\begin{aligned}
\mathcal{L}_{\mathrm{int}}^{\mathrm{SU}(3)} & =\frac{i}{4} h_{\overline{3} \overline{3}}^{9} \operatorname{tr}\left(\left(\partial_{\mu} \Phi_{[\overline{3}]}\right) \Phi_{[\overline{3}]}^{\dagger} V_{[9]}^{\mu}-\Phi_{[\overline{3}]}\left(\partial_{\mu} \Phi_{[\overline{3}]}^{\dagger}\right) V_{[9]}^{\mu}\right) \\
& +\frac{i}{4} h_{\overline{3} \overline{3}} \operatorname{tr}\left(\left(\partial_{\mu} \Phi_{[\overline{3}]}\right) \Phi_{[\overline{3}]}^{\dagger}-\Phi_{[\overline{3}]}\left(\partial_{\mu} \Phi_{[\overline{3}]}^{\dagger}\right)\right) \cdot \operatorname{tr}\left(V_{[9]}^{\mu}\right) \\
& +\frac{i}{4} h_{99}^{9} \operatorname{tr}\left(\left(\partial_{\mu} \Phi_{[9]}\right) \Phi_{[9]} V_{[9]}^{\mu}-\Phi_{[9]}\left(\partial_{\mu} \Phi_{[9]}\right) V_{[9]}^{\mu}\right) .
\end{aligned}
$$

It needs to be emphasized that the terms in (1) are not at odds with the constraints set by chiral symmetry provided the light vector mesons are coupled to matter fields via a gauge principle ${ }^{21,25}$. The latter requires a correlation of the coupling constants $h$ in (1)

$$
h_{99}^{9}=\frac{\left(m_{[9]}^{(V)}\right)^{2}}{2 g f^{2}}, \quad h_{\overline{3} \overline{3}}^{9}=2 g,
$$

with the pion decay constant $f \simeq 92 \mathrm{MeV}$. Here the universal vector coupling strength is $g \simeq 6.6$ and the mass of the light vector mesons is $m_{[9]}^{(V)}$. 
We continue with the construction of the three-point vertices involving baryon fields. A list of $\mathrm{SU}(3)$ invariant terms reads:

$$
\begin{aligned}
\mathcal{L}_{\mathrm{int}}^{\mathrm{SU}(3)} & =\frac{1}{2} g_{\overline{3} \overline{3}}^{9} \operatorname{tr}\left(\bar{B}_{[\overline{3}]} \gamma_{\mu} V_{[9]}^{\mu} B_{[\overline{3}]}\right)+\frac{1}{2} g_{\overline{3} \overline{3}} \operatorname{tr}\left(\bar{B}_{[\overline{3}]} \gamma_{\mu} B_{[\overline{3}]}\right) \operatorname{tr}\left(V_{[9]}^{\mu}\right) \\
& +\frac{1}{2} g_{66}^{9} \operatorname{tr}\left(\bar{B}_{[6]} \gamma_{\mu} V_{[9]}^{\mu} B_{[6]}\right)+\frac{1}{2} g_{66}^{1} \operatorname{tr}\left(\bar{B}_{[6]} \gamma_{\mu} B_{[6]}\right) \operatorname{tr}\left(V_{[9]}^{\mu}\right) \\
& +\frac{1}{2} g_{88}^{9-} \operatorname{tr}\left(\bar{B}_{[8]} \gamma_{\mu}\left[V_{[9]}^{\mu}, B_{[8]}\right]_{-}\right)+\frac{1}{2} g_{88}^{9+} \operatorname{tr}\left(\bar{B}_{[8]} \gamma_{\mu}\left[V_{[9]}^{\mu}, B_{[8]}\right]_{+}\right) \\
& +\frac{1}{2} g_{88}^{1} \operatorname{tr}\left(\bar{B}_{[8]} \gamma_{\mu} B_{[8]}\right) \operatorname{tr}\left(V_{[9]}^{\mu}\right) \\
& +\frac{1}{2} g_{\overline{3} 6}^{9} \operatorname{tr}\left(\bar{B}_{[\overline{3}]} \gamma_{\mu} V_{[9]}^{\mu} B_{[6]}+\bar{B}_{[6]} \gamma_{\mu} V_{[9]}^{\mu} B_{[\overline{3}]}\right) .
\end{aligned}
$$

Within the hidden local symmetry model ${ }^{25}$ chiral symmetry is recovered with

$$
g_{\overline{3} \overline{3}}^{9}=g_{66}^{9}=2 g, \quad g_{88}^{9,-}=g, \quad g_{88}^{9,+}=0, \quad g_{\overline{3} 6}^{9}=0 .
$$

It is acknowledged that chiral symmetry does not constrain the coupling constants in $(1,3)$ involving the $\mathrm{SU}(3)$ singlet part of the fields. The latter can, however, be constrained by a large- $N_{c}$ operator analysis ${ }^{27}$. At leading order in the $1 / N_{c}$ expansion the OZI rule ${ }^{28}$ is predicted. As a consequence the estimates

$$
h_{\overline{3} \overline{3}}^{1}=-g, \quad g_{\overline{3} \overline{3}}^{1}=g_{66}^{1}=0, \quad g_{88}^{1}=g,
$$

follow. We emphasize that the combination of chiral and large- $N_{c}$ constraints $(2,4,5)$ determine all coupling constants introduced in $(1,3)$.

We close this section by investigating the coupling of heavy vector mesons, $V_{[\overline{3}]}^{\mu}$ and $V_{[1]}^{\mu}$ to the meson and baryon fields. First we construct the most general SU(3) symmetric interaction terms involving the pseudoscalar fields:

$$
\begin{aligned}
\mathcal{L}_{\mathrm{int}}^{\mathrm{SU}(3)} & =\frac{i}{4} h_{\overline{3} \overline{3}}^{0} \operatorname{tr}\left(\left(\partial_{\mu} \Phi_{[\overline{3}]}\right) \Phi_{[\overline{3}]}^{\dagger} V_{[1]}^{\mu}-\Phi_{[\overline{3}]}\left(\partial_{\mu} \Phi_{[\overline{3}]}^{\dagger}\right) V_{[1]}^{\mu}\right) \\
& +\frac{i}{4} h_{9 \overline{3}}^{\overline{3}} \operatorname{tr}\left(\left(\partial_{\mu} \Phi_{[\overline{[\overline{3}}}^{\dagger}\right) \Phi_{[9]} V_{[\overline{3}]}^{\mu}-\Phi_{[9]}\left(\partial_{\mu} \Phi_{[\overline{3}]}\right) V_{[\overline{3}]}^{\dagger \mu}\right) \\
& +\frac{i}{4} h_{\overline{3} 9}^{\overline{3}} \operatorname{tr}\left(\left(\partial_{\mu} \Phi_{[9]}\right) \Phi_{[\overline{3}]} V_{[\overline{3}]}^{\dagger \mu}-\Phi_{[\overline{3}]}^{\dagger}\left(\partial_{\mu} \Phi_{[9]}\right) V_{[\overline{3}]}^{\mu}\right) \\
& +\frac{i}{4} h_{\overline{3} 0}^{\overline{3}} \operatorname{tr}\left(\left(\partial_{\mu} \Phi_{[1]}\right) \Phi_{[\overline{3}]} V_{[\overline{3}]}^{\dagger \mu}-\Phi_{[\overline{3}]}^{\dagger}\left(\partial_{\mu} \Phi_{[1]}\right) V_{[\overline{3}]}^{\mu}\right) \\
& +\frac{i}{4} h_{0 \overline{3}}^{\overline{3}} \operatorname{tr}\left(\left(\partial_{\mu} \Phi_{[\overline{[}]}^{\dagger}\right) \Phi_{[1]} V_{[\overline{3}]}^{\mu}-\Phi_{[1]}\left(\partial_{\mu} \Phi_{[\overline{3}]}\right) V_{[\overline{3}]}^{\dagger \mu}\right) \\
& +\frac{i}{4} h_{\overline{3} \overline{3}}^{\overline{3}} \operatorname{tr}\left(\Phi_{[\overline{3}]}^{\dagger} V_{[\overline{3}]}^{\mu}-\Phi_{[\overline{3}]} V_{[\overline{3}]}^{\dagger \mu}\right) \operatorname{tr}\left(\partial_{\mu} \Phi_{[9]}\right) \\
& +\frac{i}{4} h_{1 \overline{3}}^{\overline{3}} \operatorname{tr}\left(\left(\partial_{\mu} \Phi_{[\overline{3}]}\right) V_{[\overline{3}]}^{\dagger \mu}-\left(\partial_{\mu} \Phi_{[\overline{3}]}^{\dagger}\right) V_{[\overline{3}]}^{\mu}\right) \operatorname{tr}\left(\Phi_{[9]}\right),
\end{aligned}
$$


where the SU(4) symmetric gerneralization of (1) suggests the identification

$$
\begin{array}{llll}
h_{\overline{3} \overline{3}}^{0}=\sqrt{2} g, & h_{9 \overline{3}}^{\overline{3}}=2 g, & h_{\overline{3} 9}^{\overline{3}}=2 g, & \\
h_{\overline{3} 0}^{\overline{3}}=\sqrt{2} g, & h_{0 \overline{3}}^{\overline{3}}=\sqrt{2} g, & h_{\overline{3} 1}^{\overline{3}}=g, & h_{1 \overline{3}}^{\overline{3}}=g .
\end{array}
$$

The prediction of the $\mathrm{SU}(4)$-symmetric coupling constants can be tested against the decay pattern of the D-meson. From the empirical branching ratio ${ }^{26}$ we deduce $\left(h_{\overline{3} 9}^{\overline{3}}+h_{9 \overline{3}}^{\overline{3}}\right) / 4=10.4 \pm 1.4$, which is confronted with the $\mathrm{SU}(4)$ estimate $\left(h_{\overline{3} 9}^{\overline{3}}+h_{9 \overline{3}}^{\overline{3}}\right) / 4=g \simeq 6.6$. We observe a moderate $\mathrm{SU}(4)$ breaking pattern. Based on this result one may expect the relations (7) to provide magnitudes for the coupling constants reliable within a factor two.

We close this section with the construction of the most general SU(3) symmetric interaction involving baryon fields

$$
\begin{aligned}
\mathcal{L}_{\mathrm{int}}^{\mathrm{SU}(3)} & =\frac{1}{2} g_{88}^{0} \operatorname{tr}\left(\bar{B}_{[8]} \gamma_{\mu} B_{[8]} V_{[1]}^{\mu}\right)+\frac{1}{2} g_{66}^{0} \operatorname{tr}\left(\bar{B}_{[6]} \gamma_{\mu} B_{[6]} V_{[1]}^{\mu}\right) \\
& +\frac{1}{2} g_{\overline{3} \overline{3}}^{0} \operatorname{tr}\left(\bar{B}_{[\overline{3}]} \gamma_{\mu} B_{[\overline{3}]} V_{[1]}^{\mu}\right) \\
& +\frac{1}{2} g_{86}^{\overline{3}} \operatorname{tr}\left(\bar{B}_{[8]} \gamma_{\mu} B_{[6]} V_{[\overline{3}]}^{\dagger \mu}+\bar{B}_{[6]} \gamma_{\mu} B_{[8]} V_{[\overline{3}]}^{\mu}\right) \\
& +\frac{1}{2} g_{8 \overline{3}}^{\overline{3}} \operatorname{tr}\left(\bar{B}_{[8]} \gamma_{\mu} B_{[\overline{3}]} V_{[\overline{3}]}^{\dagger \mu}+\bar{B}_{[\overline{3}]} \gamma_{\mu} B_{[8]} V_{[\overline{3}]}^{\mu}\right)
\end{aligned}
$$

where a SU(4) symmetric vertex implies

$$
g_{88}^{0}=0, \quad g_{66}^{0}=\sqrt{2} g, \quad g_{\overline{3} \overline{3}}^{0}=\sqrt{2} g, \quad g_{86}^{\overline{3}}=\sqrt{2} g, \quad g_{8 \overline{3}}^{\overline{3}}=-\sqrt{6} g,
$$

Unfortunately there appears to be no way at present to check on the usefulness of the result (9). Eventually simulations of QCD on a lattice may shed some light on this issue. The precise values of the coupling constants $(7,9)$ do not affect the major results of this study. This holds as long as those coupling constants range in the region suggested by $(7,9)$ within a factor two to three.

\section{S-wave baryon resonances with zero charm}

The spectrum of $J^{P}=\frac{1}{2}^{-}$baryon resonances as generated by the t-channel vector-meson exchange interaction via coupled-channel dynamics falls into two types of states. Resonances with masses above $3 \mathrm{GeV}$ couple strongly to mesons with non-zero charm content. In the $\mathrm{SU}(3)$ limit those states form an octet and a singlet. All other states have masses below $2 \mathrm{GeV}$. In the $\mathrm{SU}(3)$ limit they group into two degenerate octets and one singlet. The presence of the heavy channels does not affect that part of the spectrum at all. This is reflected in coupling constants of those states to the heavy 
channels within the typical range of $g \sim 0.1$ (see Tabs. 1-2). We reproduce the success of previous coupled-channel computations ${ }^{15,16}$, which predicts the existence of the s-wave resonances $N(1535), \Lambda(1405), \Lambda(1670), \Xi(1690)$ unambiguously with masses and branching ratios quite compatible with empirical information. There are some quantitative differences. This is the consequence of the t-channel vector meson exchange, which, only in the $\mathrm{SU}(3)$ limit with degenerate vector meson masses, is equivalent to the Weinberg-Tomozawa interaction the computation in ${ }^{15,16}$ was based on.

Most spectacular are the resonances with hidden charm above $3 \mathrm{GeV}$. The multiplet structure of such states is readily understood. The mesons with $C=-1$ form a triplet which is scattered off the $C=+1$ baryons forming an anti-triplet or sextet. We decompose the products into irreducible tensors

$$
3 \otimes \overline{3}=1 \oplus 8, \quad 3 \otimes 6=8 \oplus 10 .
$$

The coupled channel interaction is attractive in the singlet for the triplet of baryons. Attraction in the octet sector is provided by the sextet of baryons. The resulting octet of states mixes with the $\eta^{\prime}(N, \Lambda, \Sigma, \Xi)$ and $\eta_{c}(N, \Lambda, \Sigma, \Xi)$ systems. A complicated mixing pattern arises. All together the binding energies of the crypto-exotic states are large. This is in part due to the large masses of the coupled-channel states: the kinetic energy the attractive t-channel force has to overcome is reduced.

The states are narrow as a result of the OZI rule. The mechanism is analogous to the one explaining the long life time of the $J / \Psi$-meson. We should mention, however, a caveat. It turns out that the width of the crypto-exotic states is quite sensitive to the presence of channels involving the $\eta^{\prime}$ meson. This is a natural result since the $\eta^{\prime}$ meson is closely related to the $U_{A}(1)$ anomaly giving it large gluonic components. The latter work against the OZI rule. We emphasize that switching off the t-channel exchange of charm or using the $\mathrm{SU}(4)$ estimate for the latter, strongly bound crypto-exotic states are formed. In Tabs. 1-2 the zero-charm spectrum insisting on the $\mathrm{SU}(4)$ estimates $(7,9)$ is shown in the $3 \mathrm{rd}$ and 4 th column. The mass of the crypto-exotic nucleon resonance comes at $3.33 \mathrm{GeV}$ in this case. Its width of $160 \mathrm{MeV}$ is completely dominated by the $\eta^{\prime} N$ decay. The properties of that state can be adjusted easily to be consistent with the empirical values claimed in ${ }^{6}$. The $\eta^{\prime}$ coupling strength to the open-charm mesons can be turned off by decreasing the magnitude of $h_{\overline{3} 1}^{\overline{3}}$ and $h_{1 \overline{3}}^{\overline{3}}$ by $33.3 \%$ away from their $\mathrm{SU}(4)$ values. As a result the width of the resonance is down to about $1-2 \mathrm{MeV}$. It is stressed that the masses 
Table 1. Spectrum of $J^{P}=\frac{1}{2}^{-}$baryons with charm zero. The 3rd and 4th columns follow with $\mathrm{SU}(4)$ symmetric 3-point vertices. In the 5th and 6th columns $\mathrm{SU}(4)$ breaking is introduced with $h_{\overline{3} \overline{3}} \simeq-1.19 \mathrm{~g}$ and $h_{\overline{3} 1}^{\overline{3}}=h_{1 \overline{3}}^{\overline{3}} \simeq 0.71 \mathrm{~g}$. We use $g=6.6$.

\begin{tabular}{|c|c|c|c|c|c|}
\hline$C=0: \quad(I, \quad S)$ & state & $\begin{array}{l}M_{R}[\mathrm{MeV}] \\
\Gamma_{R}[\mathrm{MeV}] \\
\end{array}$ & $\left|g_{R}\right|$ & $\begin{array}{l}M_{R}[\mathrm{MeV}] \\
\Gamma_{R}[\mathrm{MeV}] \\
\end{array}$ & $\left|g_{R}\right|$ \\
\hline \multirow{7}{*}{$\left(\frac{1}{2}, 0\right)$} & $\pi N$ & \multirow{8}{*}{$\begin{array}{c}1535 \\
95\end{array}$} & $\overline{0.3}$ & \multirow{8}{*}{$\begin{array}{c}1536 \\
94\end{array}$} & 0.3 \\
\hline & $\eta N$ & & 2.1 & & 2.1 \\
\hline & $K \Lambda$ & & 1.7 & & 1.6 \\
\hline & $K \Sigma$ & & 3.3 & & 3.3 \\
\hline & $\eta^{\prime} N$ & & 0.0 & & 0.0 \\
\hline & $\eta_{c} N$ & & 0.0 & & 0.0 \\
\hline & $\bar{D} \Lambda_{c}$ & & 0.2 & & 0.2 \\
\hline \multirow{9}{*}{$\left(\frac{1}{2}, 0\right)$} & $\bar{D} \Sigma_{c}$ & & 0.2 & & 0.2 \\
\hline & $\pi N$ & \multirow{8}{*}{$\begin{array}{c}3327 \\
156\end{array}$} & 0.1 & \multirow{8}{*}{$\begin{array}{c}3520 \\
7.3\end{array}$} & 0.07 \\
\hline & $\eta N$ & & 0.1 & & 0.11 \\
\hline & $K \Lambda$ & & 0.1 & & 0.08 \\
\hline & $K \Sigma$ & & 0.1 & & 0.08 \\
\hline & $\eta^{\prime} N$ & & 1.4 & & 0.22 \\
\hline & $\eta_{c} N$ & & 0.7 & & 1.0 \\
\hline & $\overline{\bar{D}} \Lambda_{c}$ & & 0.5 & & 0.05 \\
\hline & $\bar{D} \Sigma_{c}$ & & 5.7 & & 5.3 \\
\hline \multirow{13}{*}{$(0,-1)$} & $\pi \Sigma$ & \multirow{9}{*}{$\begin{array}{c}1413 \\
10\end{array}$} & 0.7 & \multirow{9}{*}{$\begin{array}{c}1413 \\
10\end{array}$} & 0.7 \\
\hline & $\bar{K} N$ & & 2.7 & & 2.7 \\
\hline & $\eta \Lambda$ & & 1.1 & & 1.1 \\
\hline & $K \Xi$ & & 0.1 & & 0.1 \\
\hline & $\eta^{\prime} \Lambda$ & & 0.0 & & 0.0 \\
\hline & $\eta_{c} \Lambda$ & & 0.0 & & 0.0 \\
\hline & $\overline{\bar{D}}_{s} \Lambda_{c}$ & & 0.2 & & 0.2 \\
\hline & $\bar{D} \Xi_{c}$ & & 0.0 & & 0.0 \\
\hline & $\bar{D} \Xi_{c}^{\prime}$ & & 0.0 & & 0.0 \\
\hline & $\pi \Sigma$ & \multirow{9}{*}{$\begin{array}{c}1689 \\
35\end{array}$} & $\overline{0.2}$ & \multirow{9}{*}{$\begin{array}{c}1689 \\
35\end{array}$} & 0.2 \\
\hline & $\bar{K} N$ & & 0.6 & & 0.6 \\
\hline & $\eta \Lambda$ & & 1.1 & & 1.1 \\
\hline & $K \Xi$ & & 3.6 & & 3.6 \\
\hline \multirow[t]{9}{*}{$(0,-1)$} & $\eta^{\prime} \Lambda$ & & 0.0 & & 0.0 \\
\hline & $\eta_{c} \Lambda$ & & 0.0 & & 0.0 \\
\hline & $\overline{\bar{D}}_{s} \Lambda_{c}$ & & 0.1 & & 0.1 \\
\hline & $\bar{D} \Xi_{c}$ & & 0.1 & & 0.1 \\
\hline & $\bar{D} \Xi_{c}^{\prime}$ & & 0.1 & & 0.1 \\
\hline & $\pi \Sigma$ & \multirow{9}{*}{$\begin{array}{c}3148 \\
1.0\end{array}$} & 0.04 & \multirow{9}{*}{$\begin{array}{l}3234 \\
0.57\end{array}$} & 0.04 \\
\hline & $\bar{K} N$ & & 0.03 & & 0.03 \\
\hline & $\eta \Lambda$ & & 0.03 & & 0.03 \\
\hline & $K \Xi$ & & 0.04 & & 0.04 \\
\hline \multirow[t]{5}{*}{$(0,-1)$} & $\eta^{\prime} \Lambda$ & & 0.08 & & 0.01 \\
\hline & $\eta_{c} \Lambda$ & & 0.08 & & 0.06 \\
\hline & $\bar{D}{ }_{s} \Lambda_{c}$ & & 3.2 & & 3.0 \\
\hline & $\begin{array}{l}\bar{D} \Xi_{c} \\
\bar{D} \overline{-}^{\prime}\end{array}$ & & 5.0 & & 5.0 \\
\hline & $\bar{D} \Xi_{c}^{\prime}$ & & 0.1 & & 0.01 \\
\hline
\end{tabular}

of the crypto-exotic states are not affected at all. The latter are increased most efficiently by allowing an OZI violating $\phi_{\mu} D \bar{D}$ vertex. We adjust $h \frac{1}{\overline{3} \overline{3}} \simeq-1.19 \mathrm{~g}$ and $h_{\overline{3} 1}^{\overline{3}}=h_{1 \overline{3}}^{\overline{3}} \simeq 0.71 \mathrm{~g}$ as to obtain the nucleon resonance mass and width at $3.52 \mathrm{GeV}$ and $7 \mathrm{MeV}$. For all other parameters the $\mathrm{SU}(4)$ estimates are used. The result of this choice of parameters is shown in the last two rows of Tabs. 1-2. Further crypto-exotic states, members of the aforementioned octet, are predicted at mass $3.58 \mathrm{GeV}(0,-1)$ and 3.93 
Table 2. Continuation of Tab. 1.

\begin{tabular}{|c|c|c|c|c|c|}
\hline$C=0: \quad\left(\begin{array}{ll}I, & S\end{array}\right)$ & state & $\begin{array}{l}M_{R}[\mathrm{MeV}] \\
\Gamma_{R}[\mathrm{MeV}] \\
\end{array}$ & $\left|g_{R}\right|$ & $\begin{array}{l}M_{R}[\mathrm{MeV}] \\
\Gamma_{R}[\mathrm{MeV}] \\
\end{array}$ & $\left|g_{R}\right|$ \\
\hline$(0,-1)$ & $\begin{array}{l}\pi \Sigma \\
\bar{K} N \\
\eta \Lambda \\
K \Xi \\
K \Xi \\
\eta^{\prime} \Lambda \\
\eta_{c} \Lambda \\
\bar{D} \Lambda_{c} \Lambda_{c} \\
\bar{D} \Xi_{c} \\
\bar{D} \Xi_{c}^{\prime}\end{array}$ & $\begin{array}{c}3432 \\
161\end{array}$ & $\begin{array}{l}0.1 \\
0.0 \\
0.0 \\
0.1 \\
1.3 \\
0.7 \\
0.6 \\
0.1 \\
5.6 \\
\end{array}$ & $\begin{array}{c}3581 \\
4.9\end{array}$ & $\begin{array}{c}0.06 \\
0.01 \\
0.03 \\
0.07 \\
0.20 \\
0.93 \\
0.05 \\
0.02 \\
5.3\end{array}$ \\
\hline$(1,-1)$ & $\begin{array}{l}\pi \Lambda \\
\pi \Sigma \\
\bar{K} \Sigma \\
\eta \Sigma \Sigma \\
K \Xi \\
\bar{T} \Sigma \\
\eta^{\prime} \Sigma \\
\eta_{c} \Sigma \\
\bar{D} \Xi_{c} \\
\bar{D} s \Sigma_{c} \\
\bar{D} \Xi_{c}^{\prime}\end{array}$ & $\begin{array}{l}3602 \\
227\end{array}$ & $\begin{array}{l}0.1 \\
0.1 \\
0.2 \\
0.1 \\
0.1 \\
1.5 \\
1.2 \\
0.6 \\
4.6 \\
2.9 \\
\end{array}$ & $\begin{array}{c}3930 \\
11\end{array}$ & $\begin{array}{c}0.08 \\
0.04 \\
0.12 \\
0.08 \\
0.06 \\
0.27 \\
1.8 \\
0.11 \\
3.6 \\
2.4 \\
\end{array}$ \\
\hline$\left(\frac{1}{2},-2\right)$ & $\begin{array}{l}\pi \Xi \\
\bar{K} \Lambda \\
\bar{K} \Sigma \\
\eta \Xi \\
\eta^{\prime} \Xi \\
\eta_{c} \Xi \\
\bar{D} \Xi_{s} \Xi_{c} \\
\bar{D} \\
\bar{D} \\
\bar{D} \Omega_{c}\end{array}$ & $\begin{array}{c}1644 \\
3.0\end{array}$ & $\begin{array}{l}0.1 \\
0.4 \\
2.8 \\
1.3 \\
0.0 \\
0.0 \\
0.2 \\
0.1 \\
0.0\end{array}$ & $\begin{array}{c}1644 \\
3.1\end{array}$ & $\begin{array}{l}0.1 \\
0.4 \\
2.8 \\
1.3 \\
0.0 \\
0.0 \\
0.2 \\
0.1 \\
0.0\end{array}$ \\
\hline$\left(\frac{1}{2},-2\right)$ & $\begin{array}{l}\pi \Xi \\
\bar{K} \Lambda \\
\bar{K} \Sigma \\
\eta \Xi \\
\eta \Xi \\
\eta^{\prime} \Xi \\
\eta_{c} \Xi \\
\bar{D} \Xi_{s} \Xi_{c} \\
\bar{D} s \Xi_{c}^{\prime} \\
\bar{D} \Omega_{c}\end{array}$ & $\begin{array}{c}3624 \\
204\end{array}$ & $\begin{array}{l}0.1 \\
0.1 \\
0.1 \\
0.0 \\
1.4 \\
1.0 \\
0.6 \\
3.3 \\
4.3\end{array}$ & $\begin{array}{c}3798 \\
6.0\end{array}$ & $\begin{array}{c}0.08 \\
0.04 \\
0.04 \\
0.01 \\
0.22 \\
1.2 \\
0.10 \\
2.9 \\
4.0\end{array}$ \\
\hline
\end{tabular}

$\mathrm{GeV}(1,-1)$. The multiplet is completed with a $\left(\frac{1}{2},-2\right)$ state at $3.80 \mathrm{GeV}$. The decay widths of these states center around $\sim 7 \mathrm{MeV}$. This reflects the dominance of their decays into channels involving the $\eta^{\prime}$ meson. The coupling constants to the various channels are included in Tabs. 1-2. They confirm the interpretation that the crypto-exotic states discussed above are a consequence of a strongly attractive force between the charmed mesons and the baryon sextet.

We close with a discussion of the crypto-exotic $\mathrm{SU}(3)$ singlet state, which is formed due to strong attraction in the $\left(\bar{D}_{s} \Lambda_{c}\right),\left(\bar{D} \Xi_{c}\right)$ system. Its nature is quite different as compared to the one of the octet states. This is because its coupling to the $\eta^{\prime} \Lambda$ channel is largely suppressed. Indeed its width is independent on the magnitude of $h_{\overline{3} 1}^{\overline{3}}=h_{1 \overline{3}}^{\overline{3}}$ as demonstrated in Tabs. 1-2. We identify this state with a signal claimed in the $K^{-} p$ reaction, where a 
narrow hyperon state with $3.17 \mathrm{GeV}$ mass and width smaller than $20 \mathrm{MeV}$ was seen ${ }^{5}$. Using values for the coupling constants as suggested by $\mathrm{SU}(4)$ the state has a mass and width of $3.148 \mathrm{GeV}$ and $1 \mathrm{MeV}$ (see 3rd and 4th column of Tabs. 1-2). Our favored parameter set with $h_{\overline{3} \overline{3}}^{1} \simeq-1.19 \mathrm{~g}$ and $h_{\overline{3} 1}^{\overline{3}}=h_{1 \overline{3}}^{\overline{3}} \simeq 0.71 \mathrm{~g}$ predicts a somewhat reduced binding energy.

\section{Summary}

We reviewed a coupled-channel study of s-wave baryon resonances with charm 0 . The interaction is defined by the exchange of light vector mesons in the t-channel. All relevant coupling constants are obtained from chiral and large- $N_{c}$ properties of QCD. Less relevant vertices related to the tchannel forces induced by the exchange of charmed vector mesons were estimated by applying SU(4) symmetry. Most spectacular is the prediction of narrow crypto-exotic baryons with charm zero forming below $4 \mathrm{GeV}$. Such states contain a $c \bar{c}$ pair. Their widths parameters are small due to the OZI rule, like it is the case for the $J / \Psi$ meson. We predict an octet of crypto-exotic states which decay dominantly into channels involving an $\eta^{\prime}$ meson. An even stronger bound crypto-exotic $\mathrm{SU}(3)$ singlet state is predicted to have a decay width of about $1 \mathrm{MeV}$ only. We recover the masses and widths of a crypto-exotic nucleon and hyperon resonance suggested in high statistic bubble chamber experiments ${ }^{5,6}$.

\section{References}

1. S.J. Brodsky, I. Schmidt and G.F. de Teramond, Phys. Rev. Lett. 64 (1990) 1011.

2. A.B. Kaidalov and P.E. Volkovitsky, Phys. Rev. Lett. 69 (1992) 3155.

3. C. Gobbi, D.O. Riska and N.N. Scoccola, Phys. Lett. B 296 (1992) 166.

4. L.G. Landsberg, Phys. Atom. Nucl. 57 (1994) 2127.

5. T. Amirzadeh et al., Phys. Lett. B 89 (1979) 125.

6. V.M. Karnaukhov et al., Phys. Lett. B 281 (1991) 148.

7. J. Hofmann and M.F.M. Lutz, hep-ph/0507071.

8. M.F.M. Lutz and E.E. Kolomeitsev, Nucl. Phys. A 730 (2004) 110.

9. M.F.M. Lutz and E. E. Kolomeitsev, Nucl. Phys. A 755 (2005) 29c.

10. E.E. Kolomeitsev and M.F.M. Lutz, Phys. Lett. B 585 (2004) 243.

11. J. Hofmann and M.F.M. Lutz, Nucl. Phys. A 733 (2004) 142.

12. M.F.M. Lutz and E. E. Kolomeitsev, Nucl. Phys. A 700 (2002) 193.

13. M.F.M. Lutz, Gy. Wolf and B. Friman, Nucl. Phys. A 706 (2002) 431.

14. M.F.M. Lutz and E.E. Kolomeitsev, Nucl. Phys. A 730 (2004) 392.

15. C. García-Recio, M.F.M. Lutz and J. Nieves, Phys. Lett. B 582 (2004) 49.

16. E.E. Kolomeitsev and M.F.M. Lutz, Phys. Lett. B 585 (2004) 243.

17. Ch. Hong-Mo and H. Högaasen, Z. Phys. C 7 (1980) 25. 
18. M. Rho, D.O. Riska and N.N. Scoccola, Z. Phys. A 341 (1992) 343.

19. D.-P. Min, Y. Oh, B.-Y. Park and M. Rho, Int. J. Mod. E 4 (1995) 47.

20. Y. Oh and H. Kim, Phys. Rev. D 70 (2004) 094022.

21. S. Weinberg, Phys. Rev. 166 (1968) 1568.

22. H.W. Wyld, Phys. Rev. 155 (1967) 1649.

23. R.H. Dalitz, T.C. Wong and G. Rajasekaran, Phys. Rev. 153 (1967) 1617.

24. P.B. Siegel and W. Weise, Phys. Rev. C 38 (1988) 2221.

25. M. Bando et al., Phys. Rev. Lett. 54 (1985) 1215.

26. S. Eidelman et al., Phys. Lett. B 592 (2004) 1.

27. R. Dashen, E. Jenkins and A.V. Manohar, Phys. Rev. D 49 (1994) 4713.

28. S. Okubo, Phys. Lett. 5 (1963) 165; G. Zweig, CERN Reports TH-401, TH412 (1964), unpublished; J. Iiuzuka, Prog. Theor. Part. Sci. 37 (1966) 21. 